\title{
Rapid Progression of Endogenous Endophthalmitis in Immunocompromised Patients
}

\author{
Jacqueline Yih Ling Ting로 Bin Hoo Teo², Safinaz Mohd Khialdin³ ${ }^{3}$ Mushawiahti Mustapha ${ }^{4}$ \\ 1, 2, 3, 4 Department of Ophthalmology, University Kebangsaan Malaysia Medical Centre (UKMMC), \\ Jalan Yaacob Latiff Cheras, Kuala Lumpur, Malaysia.
}

\section{INTRODUCTION}

Endophthalmitis is a severe inflammatory disease classified as either endogenous or exogenous, depending on the route of infection. The disease may be caused by trauma, intraocular surgery, infectious keratitis or other endogenous causes.1,2 Endogenous endophthalmitis develops when the infectious agent travels via the bloodstream, crosses the blood ocular barrier and proliferates within the eye. ${ }^{3}$ Endogenous endophthalmitis accounts for approximately $5-8 \%$ of all endophthalmitis cases. ${ }^{1}$ Specific predisposing factors may cause patients more at risk for rapid disease progression. ${ }^{3,4}$ Besides, source of the infection varies widely from the respiratory tract to urinary tract or liver abscess. ${ }^{5}$ We report a case series of endogenous endophthalmitis among immunocompromised patients. These cases highlighted the importance of early diagnosis with appropriate timely intervention to prevent further spread of infection and reduce the complications.

\section{PRESENTATION OF CASE}

\section{Case 1}

A 63-year-old gentleman with poorly controlled diabetes was admitted under medical care for community acquired pneumonia. He was referred to ophthalmology team for left eye preseptal swelling. He complained of one-week history of progressive painless vision loss over the left eye. Vision was 6/12 over the right eye and no perception of light over the left eye with positive relative afferent pupillary defect (RAPD). The left eyelid was swollen, erythematous and the conjunctiva was chemosed. Cornea was hazy with presence of cells in the anterior chamber and raised intraocular pressure of $35 \mathrm{mmHg}$. Furthermore, B-scan showed dense vitritis suggestive of endophthalmitis. His blood investigations showed elevated inflammatory markers with presence of leucocytosis, neutrophilia and elevated erythrocyte sedimentation rate (ESR) as well as C-reactive protein (CRP). Anterior chamber fluid and vitreous tap depicted no growth. Blood culture also came back as negative. Subsequently, bronchopulmonary lavage was done in view of the unresolved pneumonia. The lavage was the only positive culture, depicting Candida tropicalis which was sensitive to amphotericin B. Imaging including ultrasound abdomen and Computed Tomography (CT) brain and orbit were normal. He was initiated with intravitreal antifungal amphotericin B $(0.0005 \mathrm{mg}$ in $0.1 \mathrm{ml})$ with antimicrobial therapy of ceftazidime $(2 \mathrm{mg}$ in $0.1 \mathrm{ml})$ and vancomycin $(2 \mathrm{mg}$ in 0.1 ml). He was also started with topical amphotericin B $3 \%$, vancomycin 5\%, ceftazidime $5 \%$, and intravenous ceftriaxone $2 \mathrm{~g}$ twice daily and amphotericin B 15 mg once daily. However, patient was not stable and eye condition worsened. New vitreous loculations with thickened sclera and choroid noted on B scan. In addition, patient developed metabolic acidosis due to his hyperglycaemic state and also ongoing infection. Patient was counselled for early evisceration in view of progression to panophthalmitis. Patient subsequently underwent evisceration under general anaesthesia two weeks later in view of the disease progression with a poor visual prognosis. Postoperatively, patient's general condition improved tremendously.
Corresponding Author: Dr. Safinaz Mohd Khialdin Department of Ophthalmology, University Kebangsaan Malaysia Medical Centre (UKMMC) Jalan Yaacob Latiff Cheras, Kuala Lumpur, Malaysia. E-mail: drsafinaz_1978@yahoo.com.my DOI: $10.14260 / j e m d s / 2021 / 646$

How to Cite This Article:

Ting JYL, Teo BH, Khialdin SM, et al. Rapid progression of endogenous endophthalmitis in immunocompromised patients - case series. J Evolution Med Dent Sci 2021;10(36):3184-3187, DOI: $10.14260 / \mathrm{jemds} / 2021 / 646$

Submission 10-06-2021, Peer Review 03-08-2021, Acceptance 10-08-2021, Published 06-09-2021.

Copyright (C) 2021 Jacqueline Yih Ling Ting et al. This is an open access article distributed under Creative Commons Attribution License [Attribution 4.0 International (CC BY 4.0)] 

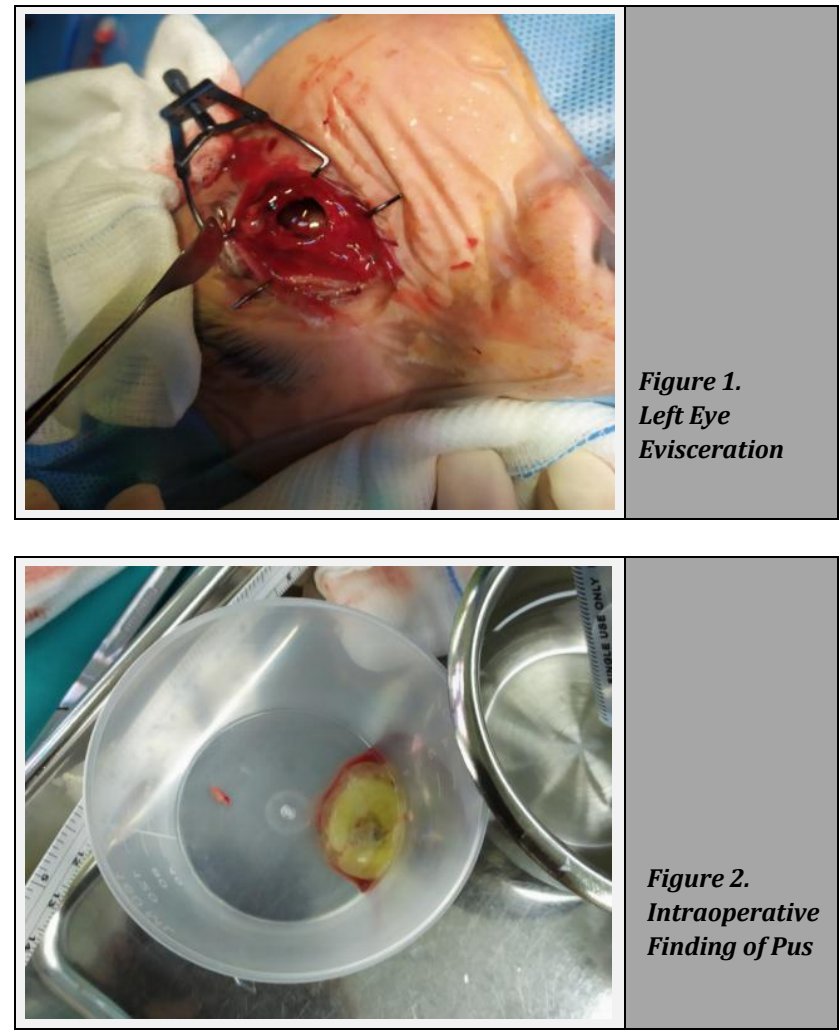

\section{Case 2}

A 47-year-old gentleman with poorly controlled diabetes presented with two weeks' history of progressive reduced vision and redness over the left eye. He presented with episodes of low grade fever and suprapubic pain. Patient denied history of ocular trauma or intraocular surgery. Systemically, he was on insulin treatment for his diabetes but was sub-optimized due to poor compliance. Ocular examination revealed hand movement vision over the left eye with positive RAPD. The left eyelid was erythematous with injected conjunctiva. There was presence of anterior chamber cells. His intraocular pressure was $19 \mathrm{mmHg}$. Extraocular movements were limited in all directions. Fundus examination revealed a dome-shaped choroidal lesion covering the macula with peripheral choroidal detachment. Meanwhile, vision over the right eye was $6 / 24$ and showed proliferative diabetic retinopathy changes. B-scan revealed a dome shaped lesion over the posterior pole with retinal detachment. Laboratory investigations showed leucocytosis with neutrophilia, highly elevated ESR and CRP. CT brain and orbit depicted inflamed retro-orbital tissue with no intracranial extension. Vitreous sample depicted no growth. His blood culture grew enterobacter sp. which was sensitive to cefepime. Thus, the patient was treated for left eye endogenous bacterial endophthalmitis with intravitreal ceftazidime ( $2 \mathrm{mg}$ in $0.1 \mathrm{ml}$ ) and vancomycin $(2 \mathrm{mg}$ in $0.1 \mathrm{ml})$. He was also started with systemic intravenous ceftriaxone $2 \mathrm{~g}$ twice daily and topical ceftazidime $5 \%$. His deranged liver and renal profile prevented the use of other antimicrobials of better penetration. His vision continued to deteriorate further to no perception to light. With continuous monitoring, the choroidal abscess eventually showed regression.

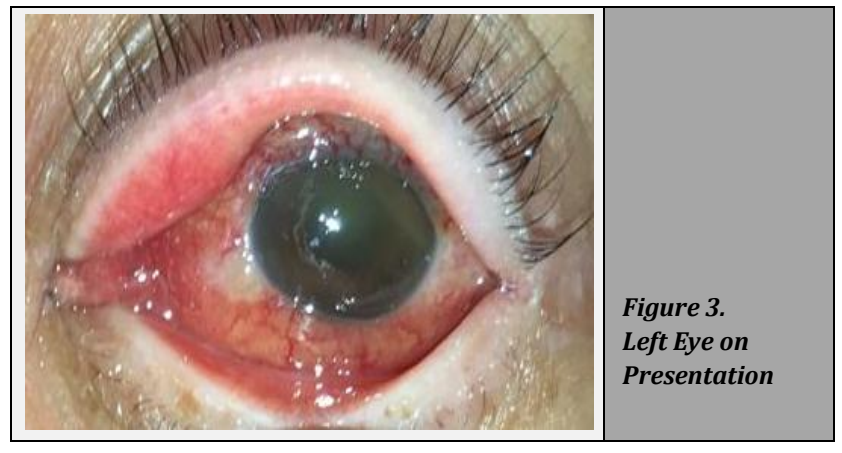

\section{Case 3}

A 45-year-old gentleman with poorly controlled diabetes was admitted for left neck abscess. He was referred to the ophthalmology unit for reduced vision over left eye. He complained of sudden onset of blurry vision in his left eye for 3 days associated with redness. He had no other systemic complaint apart from being treated for the left neck abscess. Left eye vision was 4/60 with no RAPD. There was no eyelid swelling but his conjunctiva was chemosed and injected. Anterior segment examination depicted presence of hypopyon in the anterior chamber. His intraocular pressure was normal. Fundus examination depicted vitritis with choroidal abscess. B scan revealed presence of choroidal abscess and vitreous loculation. Right eye was normal. Laboratory investigations showed leucocytosis with neutrophilia. Intravitreal tapping was attempted but vitreous depicted no growth. Incision and drainage was done over his neck abscess and culture came back with growth of Klebsiella Pneumoniae sensitive to coamoxiclav and cefuroxime. Patient was treated as left eye endogenous bacterial endophthalmitis at this stage. He was initiated with intravitreal antimicrobial therapy of ceftazidime ( $2 \mathrm{mg}$ in $0.1 \mathrm{ml}$ ) and vancomycin $(2 \mathrm{mg}$ in $0.1 \mathrm{ml}$ ) in addition to systemic dose of intravenous co-amoxiclav $1.2 \mathrm{~g}$ twice daily. Despite initiation of treatment, his condition progressed rapidly within a week. His vision worsened to perception of light and positive RAPD. Patient was referred to another hospital with vitreoretinal services. Lens aspiration and trans pars plana vitrectomy were done under general anaesthesia. Intraoperatively, there was very dense vitritis, significant amount of fibrosis with multiple area of choroidal elevations likely due to the choroidal abscess. Multiple foci of traction retinal detachment were also noted. Repeated intravitreal injection was given at the end of the surgery. Post operatively, vision became non-perception to light in all quadrants. There was no fundus view but B scan depicted remnants of loculation and choroidal detachment.

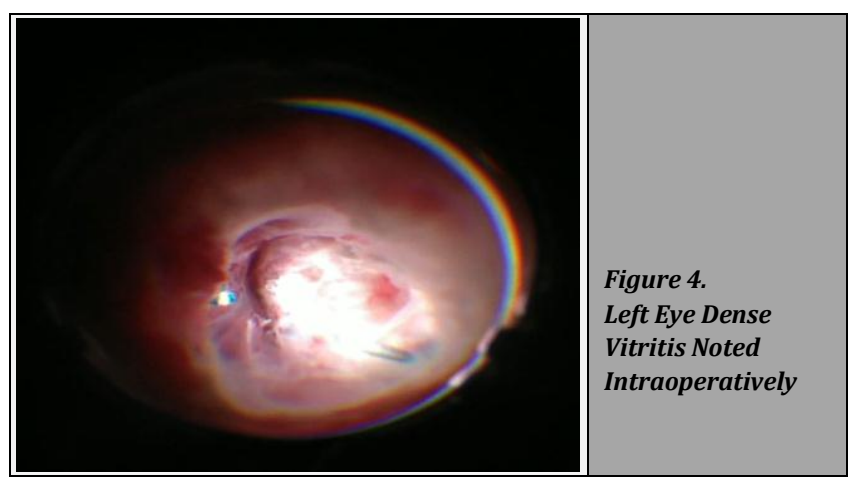




\section{Case 4}

A 39-year-old gentleman with underlying acute lymphoblastic leukaemia (ALL) and lupus nephritis was admitted for infection post brachiocephalic fistula creation. He was referred to ophthalmology team to rule out endophthalmitis in view of disseminated candidemia. His blood culture and knee aspiration fluid grew Candida Albicans which was sensitive to amphotericin B. Upon history taking, patient did not notice any reduced vision and denied other ocular symptoms. He denied history of ocular trauma or surgery. Right eye vision was $6 / 12$ while left eye vision was $6 / 6$ with no RAPD. Bilateral anterior segment was normal with normal intraocular pressure. Fundus examination over the right eye depicted choroidal lesion of one-disc diameter size at the fovea with surrounding choroiditis. Left eye fundus also depicted multiple choroidal white lesion suggestive of choroiditis. Patient was treated as bilateral fungal endogenous endophthalmitis at this stage. Intravitreal tapping was attempted, and patient was also given intravitreal amphotericin B $(0.0005 \mathrm{mg}$ in $0.1 \mathrm{ml})$ in addition to topical amphotericin B $3 \%$ and intravenous dose of $35 \mathrm{mg}$ once daily. Patient's ocular condition improved following initial intravitreal antifungal as his vision became $6 / 6$ bilaterally with resolving choroidal lesion. However, patient's poor general condition prevented further intravitreal injection.

\begin{tabular}{|c|c|c|c|c|c|c|c|}
\hline Patient & Laterality & $\begin{array}{l}\text { Initial Visual } \\
\text { Acuity }\end{array}$ & $\begin{array}{c}\text { Duration from } \\
\text { Complaint to Referral }\end{array}$ & Risk Factor & $\begin{array}{c}\text { Systemic Causative } \\
\text { Pathogen }\end{array}$ & $\begin{array}{l}\text { Cultured } \\
\text { Vitreous }\end{array}$ & Final Visual Acuity \\
\hline 1 & Left eye & NPL & 1 week & Uncontrolled Diabetes & Candida tropicalis & No growth & No light perception \\
\hline 2 & Left eye & HM & 2 weeks & Uncontrolled Diabetes & Enterobacter & No growth & No light perception \\
\hline 3 & Left eye & $4 / 60$ & 3 days & Uncontrolled Diabetes & Klebsiella Pneumoniae & No growth & Light perception \\
\hline 4 & Bilateral eyes & RE $6 / 12$ LE $6 / 6$ & $\begin{array}{l}\text { Referred without eye } \\
\text { complaints }\end{array}$ & $\begin{array}{c}\text { Acute Lymphoblastic } \\
\text { Leukaemia }\end{array}$ & Candida Albicans & No growth & $6 / 6$ \\
\hline \multicolumn{8}{|c|}{ Table 1. Summary of Clinical Information of the Patients } \\
\hline
\end{tabular}

\section{DISCUSSION}

Endogenous endophthalmitis is an intraocular infection which gains entry to the eye via haematogenous route. ${ }^{2}$ Majority of patients were immunocompromised with underlying systemic medical conditions such as concurrent renal disease, endocarditis, malignancy and ongoing immunosuppressive therapy. ${ }^{2}$ One of our patients had underlying acute lymphoblastic leukaemia (ALL) while the rest had poorly controlled diabetes. This have placed them at a higher risk of endogenous endophthalmitis. Diabetic patients have impaired response to antigens and also altered phagocytic capabilities. ${ }^{6}$ Moreover, poor glycaemic control is highly associated with impaired neutrophil bactericidal function. ${ }^{6}$ As the global epidemic of diabetes mellitus continues to expand, we would expect the incidence of endogenous endophthalmitis associated with diabetes to steadily increase in future. Chung et al. also described close association between diabetes and endogenous endophthalmitis. ${ }^{7}$

Endogenous endophthalmitis is most often caused by bacteria or fungi and the causative pathogen vary in different parts of the world. Wu ZH et al. reported that bacterial cause is responsible for $72 \%$ of endogenous endophthalmitis cases in Hong Kong8 ${ }^{8}$ while Schiedler et al. reported that fungi as a more common cause than bacteria in United States of America (USA). ${ }^{9}$ Interestingly, the incidence of gram-negative pathogen associated endophthalmitis is also on the rise in Western nations over the last two decades. ${ }^{1}$ In addition, a review in Korea reported that the commonest pathogens associated with endogenous endophthalmitis are gram negative bacteria; Klebsiella Pneumonia and Pseudomonas Aeruginosa. ${ }^{7}$ Similarly, two of the patients depicted had Klebsiella Pneumonia and enterobacter sp as the causative pathogens. The most common organism associated with endogenous fungal endophthalmitis is the yeast species Candida Albicans (87 \%) followed by Candida Tropicalis (5\%) and other mold such as aspergillus species. ${ }^{4}$ Comparing to the patients depicted above, two of them depicted fungal growth; Candida Tropicalis and Candida Albicans. Therefore, we can hypothesize that common causative pathogens in endogenous endophthalmitis among immunocompromised patients include both bacteria and fungus.

Moreover, clinical diagnosis in these patients were challenging. From the initial diagnosis of endophthalmitis, endogenous source of the infection needs to be thoroughly investigated. Diagnosis is typically made following microbiologic evidence of infection from an intraocular sample of either the aqueous or vitreous fluid ${ }^{1}$ or from extraocular source. In our patients, vitreous culture came back negative. This is most possibly due to the initiation of systemic treatment prior to vitreous sampling. Multimodal imaging including B-scan and MRI scan aid in delineating and evaluating the extent of infection. CT scans, ultrasound abdomen and chest X-ray are useful to determine the source of infection. Likewise, all these investigations were also done in the patients described to evaluate the source and extent of infection.

Treatment is generally started empirically even before source of infection is determined. Endogenous endophthalmitis often requires both local and systemic treatment. Majority of systemic antifungal and antibiotics have poor penetration of the blood ocular barrier.10,11 Drug concentrations in the vitreous are low after systemic administration. ${ }^{10}$ Hence, intravitreal injections allow achievement of higher drug concentration with longer duration. ${ }^{12}$ Vancomycin (1-2 mg / $0.1 \mathrm{~mL}$ ) and ceftazidime (2.25 mg / $0.1 \mathrm{~mL}$ ) remain the intravitreal antibiotics of choice. ${ }^{13}$ Meanwhile, for fungal cases, intravitreal voriconazole $(100 \mu \mathrm{g} / 0.1 \mathrm{ml})$ or amphotericin B (5 to $10 \mu \mathrm{g} /$ $0.1 \mathrm{ml}$ ) are given to ensure adequate level of treatment. ${ }^{13}$ Jackson et al. also suggested that combination of intravitreal and systemic treatment reduce the need for enucleation. ${ }^{1}$ However, in the three cases illustrated, the patients had rapid progression of endophthalmitis despite initiation of both systemic and intravitreal treatment. This was evidenced by clinical signs of worsening eyelid swelling, restricted extraocular movements with thickened sclera and choroid. One of the reasons could be due to late referral to the ophthalmology team. Patients were referred after one week of complaint. Infection would have worsened and more resistance to treatment. Interestingly, the fourth patient was 
referred early and patient had no initial ocular complaints. Despite having underlying malignancy and very poor general condition, his vision improved to $6 / 6$ bilaterally with only one dose of intravitreal antifungal. Thus, early referral and timely initiation of treatment is necessary to prevent progression and avoid poor visual outcomes. ${ }^{13}$

Early vitrectomy can be considered in most cases to remove the infected elements to prevent further spread of infection. ${ }^{14}$ Although the role of surgical treatment is still debatable, newer vitreoretinal technology supports early surgical intervention to remove infectious agents and inflammatory cells from vitreous cavity; increasing vitreous transparency when the infection resolves. ${ }^{15,16}$ Vitrectomy would have been considered for our patients. However, their poor general condition prevented a more aggressive surgical management and contributed to the rapid progression in the patients illustrated. This can cause further progression to devastating condition; panophthalmitis. Visual outcome of panophthalmitis is generally poor and treatment at this stage is usually aimed at lifesaving rather than vision as depicted in the first case. Approximately $24 \%$ of patients require evisceration or enucleation despite aggressive broadspectrum intravitreal and intravenous treatment. ${ }^{2}$

\section{CONCLUSIONS}

In conclusion, these cases highlight the importance of early referral of possible endogenous endophthalmitis case especially among high risk patients. Most patients were referred late which led to rapid progression and further complications as opposed to patient who was referred earlier. It is essential to establish the diagnosis in early stage and properly investigate for source of infection. Aggressive treatment aimed at both vision and lifesaving should be initiated early without delay to prevent further severe complications.

Financial or other competing interests: None.

Disclosure forms provided by the authors are available with the full text of this article at jemds.com.

\section{REFERENCES}

[1] Jackson TL, Paraskevopoulos T, Georgalas I. Systematic review of 342 cases of endogenous bacterial endophthalmitis. Surv Ophthalmol 2014;59(6):627-35.

[2] Wong JS, Chan TK, Lee HM, et al. Endogenous bacterial endophthalmitis: an East Asian experience and a reappraisal of a severe ocular affliction. Ophthalmology 2000;107(8):1483-91.

[3] Regan KA, Radhakrishnan NS, Hammer JD, et al. Endogenous endophthalmitis: yield of the diagnostic evaluation. BMC Ophthalmol 2020;20(1):138.

[4] Sridhar J, Flynn HW, Kuriyan AE, et al. Endogenous fungal endophthalmitis: risk factors, clinical features and treatment outcomes in mold and yeast infections. J Ophthalmic Inflamm Infect 2013;3(1):60.

[5] Jackson T, Eykyn S, Graham E, et al. Endogenous bacterial endophthalmitis: a 17-year prospective series and review of 267 reported cases. Surv Ophthalmol 2003;48(4):40323.

[6] Alba-Loureiro TC, Munhoz CD, Martins JO, et al. Neutrophil function and metabolism in individuals with diabetes mellitus. Braz J Med Biol Res 2007;40(8):103744.

[7] Chung KS, Kim YK, Song YG, et al. Clinical review of endogenous endophthalmitis in Korea: a 14-year review of culture positive cases of two large hospitals. Yonsei Med J 2011;52(4):630-4.

[8] Wu ZHY, Chan RPS, Luk FOJ, et al. Review of clinical features, microbiological spectrum and treatment outcomes of endogenous endophthalmitis over an 8-year period. J Ophthalmol 2012;2012:265078.

[9] Schiedler V, Scott IU, Flynn HW Jr, et al. Culture-proven endogenous endophthalmitis: clinical features and visual acuity outcomes. Am J Ophthalmol 2004;137(4):725-31.

[10] Grzybowski A, Turczynowska M, Schwartz SG, et al. The role of systemic antimicrobials in the treatment of endophthalmitis: a review and an international perspective. Ophthalmol Ther 2020;9(3):485-98.

[11] Baum J, Peyman GA, Barza M. Intravitreal administration of antibiotic in the treatment of bacterial endophthalmitis. III. Consensus. Surv Ophthalmol 1982;26(4):204-6.

[12] Radhika M, Mithal K, Bawdekar A, et al. Pharmacokinetics of intravitreal antibiotics in endophthalmitis. J Ophthalmic Inflamm Infect 2014;4:22.

[13] Spelta S, Di Zazzo A, Antonini M, et al. Does endogenous endophthalmitis need a more aggressive treatment? Ocul Immunol Inflamm 2020;1-7.

[14] Celiker H, Kazokoglu H. The role of pars plana vitrectomy in the management of fungal endogenous endophthalmitis. Eur J Ophthalmol 2020;30(1):88-93.

[15] Sheu SJ. Endophthalmitis. Korean J Ophthalmol 2017;31(4):283-9.

[16] Modjtahedi BS, Finn AP, Barb SM, et al. Characteristics and outcomes of endogenous endophthalmitis: eight-year experience at a tertiary care center. Ophthalmol Retina 2019;3(1):61-72. 